\title{
ORDRE DES DÉCRETS DIVINS, HIÉRARCHIE DES DROITS HUMAINS
}

\author{
François LAPLANCHE
}

RÉsumé : Jusqu'au Xvi ${ }^{\mathrm{e}}$ siècle, l'emploi de la force publique au service de l'unité religieuse semble être une conséquence du zèle pour le salut d'autrui. Pour parvenir à la concorde civile, dans les nations où s'affrontaient des confessions différentes, il a fallu trouver un fondement théologique à la reconnaissance juridique de la liberté de conscience et de culte. Les théologiens protestants de l'académie de Saumur fondée par Philippe Duplessis-Mornay, négociateur de l'édit de Nantes, ont cherché ce fondement du côté d'une théologie de la création. Dieu a créé l'homme pour vivre paisiblement en compagnie de ses semblables et le drame de la chute originelle, bien qu'il ait entrainé pour sa réparation une révélation de Dieu protégée par l'Église, n'a pas détruit cet ordre tout à fait primitif. «Nous sommes hommes, écrit Amyraut, avant que d'être chrétiens. »

Mots-CLÉs: Duplessis-Mornay, liberté de conscience, nature humaine, théologie de la création, Saumur.

ABSTRACT : Until the XVI ${ }^{\text {th }}$ century, the use of public force in the service of religious unity seems to be a consequence of a zeal for the salvation of others. In order to reach civil peace, in the nations where different creeds confronted each other, it was necessary to find a theological foundation for the legal recognition of a freedom of conscience and of religion. The Protestant theologians of the Academy of Saumur, founded by Philippe Duplessis-Mornay, a negotiator of the Edict of Nantes, sought this foundation in a theology of creation. God created man to live peacefully in the company of his fellow-men and the drama of original sin, although it led for its reparation to a revelation from God, protected by the Church, did not destroy this primary order. "We are men, writes Amyraut, before being Christians. »

KEYWORDS : Duplessis-Mornay, freedom of conscience, human nature, theology of creation, Saumur.

Revue de synthèse : $5^{\mathrm{e}}$ série, année 2005/1, p. 51-65. 
ZuSAMMENFASSUNG : Bis zum 16. Jahrhundert scheint der Einsatz von staatlicher Gewalt im Dienst der religiösen Einheit aus dem Eifer für das Heil anderer Menschen zu folgen. Um in Nationen, in denen sich unterschiedliche Konfessionen gegenüberstanden, ein harmonisches Zusammenleben der Bürger zu ermöglichen, mußte ein theologisches Fundament gefunden werden, das die juristische Anerkennung der Gewissensfreiheit und des protestantischen Gottesdienstes ermöglichte. Die protestantischen Theologen der von Philippe Duplessis-Mornay (er hatte das Edikt von Nantes ausgehandelt) gegründeten Akademie von Saumur suchten dieses Fundament in einer Theologie der Schöpfung. Danach hat Gott den Menschen erschaffen, damit er friedlich mit seinesgleichen zusammenlebt, und auch das Drama des Sündenfalls hat diese ganz ursprüngliche Ordnung nicht zerstört, obwohl zu dessen Heilung eine von der Kirche beschützte Offenbarung Gottes erforderlich ist. "Wir sind Menschen, bevor wir Christen sind», schrieb Amyraut.

StICHWÖRTER: Duplessis-Mornay, Gewissensfreiheit, menschliche Natur, Theologie der Schöpfung, Saumur.

François LaPLANCHE, né en 1928, est directeur de recherche honoraire au Centre national de la recherche scientifique. Spécialiste de l'histoire culturelle du christianisme à l'époque moderne et contemporaine, ses principales publications sont : L'Écriture, le sacré et l'histoire (Amsterdam/Maarssen, Academic Publishers Associated/Holland University Press, 1986) et $L a$ Bible en France entre mythe et critique, XVI'-XIX ${ }^{e}$ siècle (Paris, Albin Michel, "L'Évolution de l'humanité », 1994); de plus, il a dirigé le volume IX du Dictionnaire du monde religieux dans la France contemporaine: Les sciences religieuses (Paris, Beauchesne, 1996) et collaboré aux volumes VIII, IX et XIV de l'Histoire du christianisme (Paris, Desclée, 1992, 1997 et 2000).

Adresse : 12, square des Caléides, apt 55, F-49000 Angers.

Courrier électronique : françois.laplanche@wanadoo.fr 
L'interrogation sur les mutations théoriques du champ politique requises par la négociation et l'application de l'édit de Nantes m'a amené à privilégier deux théologiens protestants de Saumur. Philippe Duplessis-Mornay a été étroitement mêlé à la rédaction de l'édit de Nantes. Moyse Amyraut a profondément réfléchi aux conditions théoriques de pérennisation de l'Édit, une fois que, par la paix d'Alès, les protestants eurent perdu leurs places fortes, et, par là, le moyen d'exercer sur le roi une pression militaire. Sans revenir sur le détail des événements amplement présentés dans le gros numéro du Bulletin de la Société de l'histoire du protestantisme français de janvier 1998, je voudrais examiner la réflexion de fond menée par le gouverneur de Saumur sur le problème de la concorde civile, terme préférable à celui de tolérance (j'expliquerai pourquoi). Ensuite, je me propose de montrer comment Amyraut, professeur de théologie à l'académie protestante de Saumur, a cherché à construire sur de solides fondements théologiques cette théorie de la coexistence pacifique des confessions religieuses : une hiérarchie des droits humains, dont l'affirmation est hardie en cet âge de convictions religieuses ardentes, opère un choix théologique très net et agence à sa façon l'ordre des décrets divins, selon une démarche assez anthropomorphique à nos yeux, mais familière au Moyen Âge finissant et à la dogmatique calviniste.

\section{LA CONTRIBUTION DE MORNAY À LA DOCTRINE DE LA CONCORDE CIVILE}

C'est de 1595 à 1598 que Mornay, ami personnel du roi Henri IV, a joué son rôle d'intermédiaire entre les assemblées du parti protestant et le souverain. Celui-ci l'avait prié de ne pas s'éloigner de Saumur pendant ces difficiles négociations. En effet, les assemblées protestantes se tiennent à Saumur (1595), Loudun (avril-octobre 1596), Vendôme (novembre 1596février 1597), de nouveau à Saumur (mars-mai 1597), puis à Châtellerault (à partir de juin 1597). La confiance qui lui est témoignée de part et d'autre s'explique certainement par les qualités de caractère du gouverneur prompt à distinguer les exigences de la conscience protestante et les turbulences de l'agitation nobiliaire. Mais il y a plus. Dans toute grande crise religieuse ou politique, la passion des partis en présence se nourrit d'une argumentation qu'il s'agit de désarticuler pour repartir sur d'autres bases.

Mornay s'était préparé à son rôle de conciliateur en accomplissant plusieurs missions aux Pays-Bas. Constatant la division religieuse des pro- 
vinces insurgées contre le roi d'Espagne, il en avait tiré la conclusion qu'aucune action politique commune ne pouvait être envisagée sans un accord sur la religion, qui supposait un respect réciproque des catholiques et des calvinistes. Ceux-ci ne pouvaient demander la liberté de conscience et de culte s'ils ne l'accordaient pas à l'autre parti. La méfiance des catholiques ne s'expliquait que trop bien, selon Mornay, par les violences qu'ils avaient subies aux Pays-Bas :

«Ceux qui ont abattu les images, chassé la religion romaine et saisi les biens ecclésiastiques, partout où ils ont pu étendre leurs mains, ont été cause que les catholiques romains sont entrés en altération, jugeant par ces commencements que, partout où ceux de la Religion réformée seraient supportés, ils voudraient faire le semblable ${ }^{1}$.»

Pour Mornay, le mal causé par ce genre de violences constitue un état de fait. Il ne nie pas qu'un prince puisse imposer l'unité de culte sur un territoire, mais il ne peut le faire sans violenter les consciences qu'au cas où l'unanimité religieuse est acceptée de tous. Comme tel n'est pas le cas ni aux Pays-Bas ni en France, il faut trouver les voies d'un accord prenant acte des désaccords. Sur quelle base l'établir? Sur la base de la concorde civile. Dans un texte antérieur, la Remontrance aux États de Blois pour la paix (1576), Mornay avait insisté sur la triple communauté existant entre catholiques et réformés :

«Considérons donc que nous sommes tous hommes, tous chrestiens, tous François, tous amateurs de nous-mesmes, de l'Église, de la patrie, croyant en ung Dieu, confessant ung Christ, désirans une réformation en cet Estat; comme hommes, aimons; comme chrestiens, enseignons ; comme Français supportons les ungs les autres ${ }^{2}$.»

Ultérieurement, Mornay développera cette réflexion sur la triple communauté qui unit les « régnicoles », en insistant sur tel aspect ou sur tel autre, selon la conjoncture.

Quand Henri de Navarre est devenu prétendant légitime à la couronne de France par la mort du duc d'Anjou (10 juin 1584), Mornay s'efforce principalement de rassurer les catholiques sur l'entière liberté de conscience et de culte qui leur sera laissée et de légitimer l'accession au trône d'un roi protestant, c'est-à-dire, selon les catholiques, hérétique. Après la mort du roi Henri III, Henri de Navarre, qui se voit placé devant l'opposition de la

1. LeCLER, 1955, vol. II, p. 182.

2. Duplessis-Mornay, 1824-1825, vol. II, p. 77. 
Ligue à son accession au trône, est prudent vis-à-vis des protestants. Il se contente (édit de Mantes, 24 juillet 1591) de révoquer les édits de la Ligue de 1585 et 1589, ce qui remet en vigueur l'édit de Beaulieu de 1577. Pour Mornay cette mesure n'est pas suffisante. Son inquiétude augmente après l'abjuration de Saint-Denis (25 juillet 1593) ${ }^{3}$. Cette conversion a choqué Mornay. Elle ne s'est pas produite au terme du processus qu'il avait maintes fois suggéré : la convocation $\mathrm{d}^{\prime}$ ' un libre et saint Concile général », ou à défaut national, qui entendrait l'argumentation doctrinale des deux parties, ce que n'a pas fait le concile de Trente, qui a refusé d'admettre les protestants en son sein. L'abjuration de Saint-Denis offre un tout autre cas de figure : le roi s'est rangé sans réticence ni condition à la foi romaine et il en accepte toutes les excroissances pernicieuses, à savoir culte des saints, purgatoire, indulgences. Quand il a remis en vigueur l'édit de 1577, il l'a fait sans écouter les doléances de ses sujets protestants, partout en butte à l'hostilité des parlements ou des ligueurs. C'est dans cette conjoncture que Mornay le prie d'établir la paix de religion sur des bases plus fermes et participe activement aux négociations qui précèdent l'édit de Nantes. À cette période, l'action de Mornay est certainement marquée par une certaine tristesse et la fin du grand rêve protestant d'établir au royaume de France le «vrai Évangile». Il compose maintenant avec la réalité et cherche à obtenir tout ce qui est possible, en évitant la prolongation de la pression armée des forces protestantes.

Quelle que soit la conjoncture politique, la pensée de Mornay s'articule fermement autour de certains principes qu'il maintient en mettant en avant plutôt l'un que l'autre, selon l'opportunité. Ses principes, recueillis à travers ses nombreuses déclarations, mémoires et missives peuvent être ramenés, me semble-t-il, à cinq :

$1^{\circ}$ L'unité religieuse du royaume serait certes préférable, et il ne faut pas désespérer de l'obtenir par la réunion d'un libre concile, mais elle ne peut s'obtenir par le forcement des consciences. Cette violence est contraire à la dignité de la conscience et l'expérience d'un demi-siècle montre de plus son inefficacité.

$2^{\circ}$ L'unité nationale doit être l'objectif de tous les «bons François», expression qui revient souvent sous la plume de Mornay. La Ligue, en faisant appel à l'argent et aux armes du roi d'Espagne pour empêcher l'accession d'Henri de Navarre au trône de France, s'oppose directement à cet objectif. Il est vrai que les protestants ont pu chercher à obtenir des secours des Anglais, des Hollandais ou des princes allemands, mais ils n'ont pas fait ce choix les premiers.

$3^{\circ}$ L'appartenance religieuse ne change pas la condition juridique des sujets du roi. Mornay n'emploie pas le mot de droit naturel comme le fera

3. Duplessis-Mornay, 1824-1825, vol. V, p. 538. 
Amyraut, mais il entretient des pensées voisines en faisant valoir que la qualité de protestant ne modifie pas les titres de propriété d'un sujet du roi. Si ce raisonnement juridique tient debout, pourquoi Henri de Navarre perdrait-il son droit légitime à la succession de la couronne de France ? Il n'est pas hérétique, puisqu'il regarde la Bible comme la Parole de Dieu, qu'il accepte les symboles de foi, les décisions des quatre conciles généraux des $\mathrm{IV}^{\mathrm{e}}$ et $\mathrm{V}^{\mathrm{e}}$ siècles, et que, pour le reste, il est prêt à accepter les décisions d'un libre concile. Le déposséder de ses droits à la couronne de France, pour motif de religion, constituerait donc une injustice caractérisée.

$4^{\circ}$ Puisque les deux religions peuvent coexister dans une famille, dans une ville, dans une armée qui emploie des mercenaires de confession autre que celle du prince, pourquoi cette coexistence ne pourrait-elle pas être généralisée? «La messe et le presche ont seu s'accorder parmi leurs armes [...] pourquoi moins en paix publique, voisin à voisin, citoyen à citoyen, nés pour s'entreconserver, s'entresupporter et s'entr'instruire. »

$5^{\circ}$ La réalisation de la paix de religion, si légitimes qu'en soient les bases ainsi dégagées, ne peut advenir que moyennant le renoncement aux intérêts particuliers et la soumission à la volonté du roi. C'est celle-ci qui se trouve garante des trois objectifs précédemment définis : l'unité nationale, la justice entre les sujets, la concorde civile. Tacitement, cette définition de la fonction royale se soustrait évidemment à la définition par la fonction religieuse, au moins sous sa forme traditionnelle: le service de la vraie foi contre l'hérétique. Mornay maintient bien la fonction religieuse du prince, mais au moyen d'un glissement : en favorisant la guerre plutôt que la paix, le souverain favorise en fait l'irréligion et le crime, qui sont les fruits naturels de la guerre. Le roi ne doit cesser de veiller à ce que Dieu soit reconnu et servi, mais selon la forme de culte que reconnaît la conscience de chacun.

Cette définition de la fonction royale vaut aussi condamnation du déguisement des intérêts nobiliaires sous le masque de la religion. De ce point de vue, Mornay est l'adversaire des grands féodaux, qu'ils soient catholiques ou protestants.

Si l'action politique de Mornay s'inscrit avec persévérance dans le cadre d'une défense mesurée des libertés protestantes, jusqu'à quel point peut-on voir en lui un théologien de la tolérance religieuse? J'avais employé le mot dans des travaux antérieurs. Mais peut-être sans précautions suffisantes. En effet, Mornay pourrait apparaître d'un autre point de vue comme un champion de l'intolérance protestante. Avant comme après l'édit de Nantes, il attaque violemment la papauté et les pratiques catholiques dans le Traité de l'eucharistie (1598) et Le Mystère d'iniquité (1611). Le premier de ces ouvrages reprend toutes les critiques accumulées par un demi-siècle de controverse contre l'invasion du catholicisme par les rites païens. Le 
second synthétise les arguments dont use la controverse protestante pour assimiler le pape à l'Antéchrist et s'ouvre par un frontispice insultant pour la papauté, où le pontife romain est identifié à la Bête monstrueuse du chapitre XIII de l'Apocalypse ${ }^{4}$. Ce livre valut à son auteur quelques difficultés. Quant au Traité de l'eucharistie, on sait qu'il fut l'occasion de la célèbre conférence de Fontainebleau (1600) entre Mornay et le cardinal Du Perron.

Il parait donc certain que Mornay n'est pas un adepte de la tolérance religieuse, au sens où la religion de l'autre est regardée comme un chemin possible vers la vérité et en suivant lequel on peut faire son salut. Cette tolérance s'appelle au XVII ${ }^{\mathrm{e}}$ siècle la "tolérance ecclésiastique » à distinguer de la tolérance civile, cette dernière étant la licence donnée par un prince à des sujets de confession autre que la sienne de vaquer librement à leur culte. Faut-il préférer le terme de «concorde civile» à celui de «tolérance civile », en ce qui concerne Mornay, comme inclinent à le penser de jeunes historiens contemporains? Oui, et cela pour deux raisons. La première d'entre elles est que la paix de religion n'est pour Mornay qu'un pis-aller. L'abjuration d'Henri IV l'a consterné, comme je l'ai dit. Elle stoppait net l'espoir protestant de voir la victoire en France du véritable Évangile. En second lieu, la doctrine médiévale de la souveraineté du pape sur les rois de la terre et de son droit à les déposer heurtait la doctrine protestante de l'institution des rois par Dieu lui-même. Certes, l'affirmation du droit divin des rois était tenue aussi par l'Église gallicane, mais en fin de compte, ce n'étaient point les évêques français qui avaient absous Henri IV, mais le pape Clément VIII, et l'une des conditions de l'absolution était le rétablissement de l'unité de religion dans le royaume. Pouvait-on tolérer alors une religion qui ne tolérait rien en dehors d'elle ${ }^{5}$ ? Les catholiques, de leur côté, considéraient le protestantisme comme un ferment de sédition, puisque, selon ses meilleurs théologiens, comme Théodore de Bèze, le peuple n'était tenu d'obéir au roi que si le roi obéissait à Dieu, c'est-à-dire se conformait strictement aux interdictions bibliques concernant l'extermination de l'idolâtrie. Des deux côtés, donc, la tolérance de la religion de l'autre était limitée par le coefficient de nuisance politique qui lui était attribué, non sans d'excellentes raisons. Une pleine tolérance aurait exigé comme condition préalable d'un côté le renoncement aux prétentions temporelles de la papauté, de l'autre la reconnaissance de la légitimité inconditionnelle du souverain. C'est bien pourquoi la théologie politique des protestants français, au moins jusqu'aux années 1660, va insister d'une part sur le droit divin des rois, ce qui renvoie dos-à-dos ultramontains et «monarchomaques », et de l'autre sur la séparation entre les devoirs du

4. Laplanche, 1986, p. 171.

5. Mousnier, 1964, p. 100. 
souverain comme détenteur de la puissance politique et comme adepte, « en son particulier», d'une confession religieuse déterminée. Cette réflexion est particulièrement développée dans l'œuvre du théologien saumurois Moyse Amyraut (1596-1664).

L'effort spéculatif déployé par Amyraut (1596-1664) doit, pour être compris, être replacé dans son contexte politique et théologique. Le contexte politique tient à la fragilité de la situation des protestants au royaume du roi très-chrétien. Quoi qu'en aient dit ou pensé les huguenots, dans les décennies qui précèdent la Révocation, la qualification de «perpétuel et irrévocable » lisible dans le préambule de l'édit de Nantes ne constituait pas une garantie suffisante. Je citerai ici une historienne protestante contemporaine, Marianne Carbonier-Burkard. Après avoir rappelé que la formule se trouve dans les préambules de la plupart des édits de pacification qui ont précédé celui de Nantes, cependant régulièrement annulés par le dernier en date, elle ajoute : «Elle [la formule] indique une décision mûrement réfléchie, non circonstancielle, destinée à s'inscrire dans une durée non limitée. Elle n'exclut pas pour autant la possibilité de sa caducité, ni de sa révocation par un autre édit procédant également d'une mûre réflexion ${ }^{6}$. » D'ailleurs, les événements eux-mêmes qui s'étaient déroulés de 1620 à 1629, au moment donc de la jeunesse d'Amyraut, étaient très significatifs. En mai 1621, Louis XIII, conduisant son armée vers le SudOuest, avait destitué Duplessis-Mornay de son gouvernement de Saumur et le château avait été pillé. Les différents traités entre le Roi et les chefs protestants, de la paix de Montpellier à la paix d'Alès, supprimèrent les privilèges politiques concédés aux huguenots puisqu'au bout de dix ans de guerres, ils ne possédaient plus de places fortes. Par la suite, Richelieu conçut un vaste plan de réunion qui, au moyen de quelques concessions accordées aux protestants, aurait abouti à la constitution d'une sorte de patriarcat gallican ${ }^{7}$. Dans ce dessein, il envoya des émissaires secrets chargés de gagner les pasteurs un par un. C'est un jésuite nommé Étienne Audebert qui vint à Saumur ${ }^{8}$. Nous savons par une note du Dictionnaire de Bayle quelles étaient les concessions proposées aux protestants : on renoncerait au culte des saints, au purgatoire, à la doctrine du mérite des œuvres ; on limiterait le pouvoir du pape en le menaçant de créer un patriarcat auto-

6. Carbonier-Burkard, 1998, p. 92.

7. Laplanche, 1986 , p. 22-23.

8. Bots, 1978 , p. 35 . 
nome; on accorderait l'usage de la coupe aux laïcs ${ }^{9}$. Cette visite doit se situer dans la seconde moitié de l'année 1641. Elle témoigne de l'incessante pression exercée sur les protestants au temps de l'édit de Nantes. Cependant, ceux-ci n'étaient pas au bout de leurs peines, même à l'époque où ils jouissaient encore d'une relative tranquillité, c'est-à-dire avant l'avènement personnel de Louis XIV. En effet, comme l'écrit une autre historienne protestante, Élisabeth Labrousse, « un événement d'une importance capitale allait, par contrecoup, causer aux réformés français un incalculable préjudice : la Révolution d'Angleterre et son aboutissement, l'exécution de Charles $I^{\text {er }}$ le 9 février $1649^{10}$ ». De nombreux écrits de pasteurs français virent alors le jour pour blâmer ce régicide qui les embarrassait fort. Amyraut, en particulier, publia en 1650 un Discours de la souveraineté des roys. Il convient de mentionner aussi les ouvrages suivants : Apologie pour ceux de la Religion (Saumur, 1647), Six livres de la vocation des pasteurs (Saumur, 1648), Du gouvernement de l'Église (Saumur, 1653), et la longue préface latine de la Paraphrasis in Psalmos Davidis (Saumur, 1662), enfin, La Morale chrestienne (Saumur, 1652-1660, 6 vol.).

Pour bien comprendre la position d'Amyraut, il ne fallait pas seulement restituer brièvement le contexte politique de celle-ci, il convient aussi d'évoquer le contexte théologique. En effet, les débats sur la tolérance du $\mathrm{XVI}^{\mathrm{e}}$ au XVIII ${ }^{\mathrm{e}}$ siècle sont embrouillés. Le combat en faveur de la tolérance civile des cultes développe trois types d'argumentation qui peuvent d'ailleurs s'entrecroiser chez le même auteur.

$1^{\circ}$ Le principal théoricien de la tolérance au $\mathrm{XvI}^{\mathrm{e}}$ siècle, Sébastien Castellion (1515-1563), qui s'éleva à Genève contre le supplice de Michel Servet (27 octobre 1553), insiste sur la douceur évangélique, sur le caractère nonviolent du règne du Christ. Mais il ajoute à cette argumentation une claire note de relativisme. L'Écriture étant fort obscure, diverses doctrines peuvent en être tirées et seul ce qui est démontrable par la raison peut être imposé par la loi : l'existence d'un Dieu provident et rémunérateur, la force obligatoire du commandement de l'amour : «Ne fais pas à autrui ce que tu ne voudrais pas qu'on te fasse à toi-même. » Cette idée d'un accord possible autour d'un credo minimum sera développée aux Pays-Bas et en Angleterre au xvII ${ }^{\mathrm{e}}$ siècle ${ }^{11}$. Elle s'exprimera de façon radicale dans le Traité théologico-politique de Spinoza (1670), dans lequel il est soutenu que la Bible ne contenant pas de vérité spéculative, mais seulement des exhortations à la justice et à la piété, l'État ne peut employer sa puissance à autre chose qu'à favoriser la moralité publique.

9. Bots, 1978 , p. 35 .

10. Labrousse, 1985 , p. 39 sq.

11. LAGRÉE, 1989 et 1991. 
$2^{\circ}$ Dans l'aire culturelle anglo-saxonne, se développe une argumentation en faveur de la qualité de la foi. Toute pression en matière religieuse, fût-ce la coutume de baptiser les enfants sans prendre leur avis, ne peut qu'engendrer une chrétienté de médiocres et de tièdes. L'Église ne peut se recruter valablement que sur la base d'une libre décision relevant de la volonté de chacun. John Locke, vers la fin du XVII ${ }^{\mathrm{e}}$ siècle, résume clairement un siècle de réflexion anglaise sur ce point et la naissance des colonies d'Amérique portera cette marque d'origine ${ }^{12}$.

$3^{\circ}$ Les cruautés et les excès commis au nom de la religion poussent des magistrats et des juristes, doués d'une sensibilité commune en dépit des différences nationales, à contester toute forme de tutelle du religieux sur le politique. Savants en droit romain, ces légistes estiment que la religion doit être en l'État, et non l'inverse. Beaucoup d'entre eux estiment d'ailleurs que toute religion est bonne qui conforme ses préceptes à ceux de la morale naturelle et renforce ainsi l'État, en prescrivant l'exercice de la justice et de la charité entre citoyens d'une même contrée. Trop dangereuse pour être laissée entre les mains des prêtres ou des pasteurs, la religion, pour ces légistes, ne peut s'exercer que dans les limites qui lui sont prescrites par l'État souverain, qu'il soit par ailleurs républicain ou monarchique. Contre cette sorte de gens, Amyraut écrit en 1631 son Traité des religions contre ceux qui les estiment toutes indifférentes ${ }^{13}$. Ce livre parut à l'époque important : il fut réédité en 1652, traduit en anglais et en allemand (deux éditions allemandes).

Après avoir rappelé le contexte politique et théologique de la doctrine d'Amyraut, il convient de la présenter brièvement en insistant sur deux points également importants :

$1^{\circ}$ Le théologien de Saumur ne se lasse pas de rappeler l'origine divine du pouvoir royal, qui se trouve mis ainsi hors de toute contestation. Dans son Apologie pour ceux de la religion. Sur les sujets d'aversion que plusieurs pensent avoir contre leurs Personnes et leur Créance, publiée à Saumur en 1647 et 1648, il se tourne vers l'histoire du Xvi siècle en essayant de disculper les chefs protestants de toute entreprise séditieuse envers le roi. Certaines prises d'armes ne furent que des conflits entre des maisons rivales. D'autres peuvent sinon se justifier absolument du moins s'excuser du fait des violences subies par les protestants en des moments de faiblesse de l'autorité royale : le roi étant en bas âge, les adversaires des protestants en profitèrent pour les opprimer cruellement. Les guerres entreprises sous

12. LOCKE, 1980.

13. Thuau, 1966, p. 45, 213, $321 s q$. et 342. 
le règne de Louis XIII ne le furent pas par l'ensemble des protestants. Amyraut pouvait l'écrire, car en 1625 le consistoire de Paris et le synode provincial d'Île-de-France avaient désavoué la prise d'armes de Rohan et de Soubise ${ }^{14}$. De façon plus imprudente, sans doute, il avait aussi blâmé à mots couverts le soulèvement de La Rochelle et cette insinuation lui valut une longue dispute avec les Rochelais ${ }^{15}$. On notera que cette distinction entre la revendication pour la liberté de conscience et le droit à la sédition sera précisément la constante doctrine des Églises protestantes dites du désert au XVIII siècle, une fois passé l'épisode camisard.

L'exécution du roi d'Angleterre offrit à Amyraut l'occasion de développer la justification théologique de l'obéissance absolue due aux rois. Dans le Discours de la souveraineté des roys, déjà cité (supra, p. 59), il s'appuie sur l'Ancien Testament pour montrer que l'onction divine conférait aux rois d'Israël un privilège d'inviolabilité. L'institution directe des rois par le pouvoir divin demeurait une réalité de la Nouvelle Alliance et le théologien de Saumur balayait avec irritation l'exégèse biblique au moyen de laquelle les Indépendants d'Angleterre prétendaient justifier leur forfait. Un certain nombre d'historiens se sont scandalisés de ce qu'ils considèrent, non sans quelque anachronisme, comme de la flagornerie. Trompés par l'amalgame pratiqué au XVIII ${ }^{\mathrm{e}}$ siècle entre l'absolutisme monarchique et l'intolérance religieuse, ils ne voient pas que deux siècles plus tôt, c'est la puissance du roi qui, en France au moins, impose le silence aux factions religieuses en présence. Le discours adressé par Henri IV au parlement de Paris pour l'enregistrement de «son » édit est fort clair sur ce point ${ }^{16}$.

$2^{\circ}$ En faveur de la pérennisation de l'édit de Nantes, Amyraut développe une triple argumentation :

\section{Une argumentation éthico-juridique}

De même que Dieu, le Roi des rois, s'oblige lui-même à tenir ses promesses, ainsi le roi de France s'est engagé à maintenir l'édit porté par son père. L'obligation de tenir sa parole vaut en toutes circonstances. Même quand un prince chrétien a donné sa parole à un infidèle, il doit faire honneur à son engagement, fût-ce au prix d'un détriment temporel ${ }^{17}$. Il s'agit là d'un principe de droit naturel, qu'aucune autorité ne peut se permettre de transgresser. De plus, l'Édit ayant assuré par sa mise en application un demi-siècle de paix religieuse ne peut plus être remis en question, sous peine de troubles graves. Tel est le sens de la réponse que, selon Pierre

14. Pannier, 1931, vol. I, p. 256-259.

15. LAPLANCHE, 1955 , p. $63-65$.

16. Garrisson, 1998 , p. 326-328.

17. Laplanche, 1986, p. 391. 
Bayle, fort bien renseigné sur l'histoire du protestantisme français, Amyraut fit à Mazarin alors que le cardinal l'entretenait à Saumur durant la Fronde, en mars 1652. Ces entretiens devaient déboucher sur la déclaration de Saint-Germain, autorisant le rétablissement du culte protestant dans tous les lieux où des arrêts l'avaient supprimé depuis l'édit de Nantes. Mazarin fait allusion à l'argument des catholiques zélés qui assurent que l'édit de Nantes est sans valeur puisque le roi l'a concédé aux protestants qui refusaient d'aider le roi en difficulté devant Amiens. Promulgué sous la contrainte, l'acte royal était-il bien irrévocable?

«Ils parlèrent de l'édit de Nantes, raconte Bayle ${ }^{18}$, et sur ce que M. Amyraut interrogé si Henri IV avoit été dans l'obligation de le donner, avoit répondu qu'oui, mais que, quand même ç'auroit été une grâce au commencement, l'observation en seroit aujourd'hui une chose nécessaire, le Cardinal lui dit qu'il avoit raison, et lui cita cette maxime du droit : Quod initio fuit voluntatis, ex post facto fit necessitatis. »

Cette réflexion sur le droit naturel est reprise plus amplement par Amyraut dans les ouvrages cités plus haut.

\section{Une argumentation scripturaire}

Comme l'intolérance civile s'appuyait sur des textes de l'Ancien Testament (par exemple, les chapitres XIII, XVIII et xx du Deutéronome), Amyraut s'efforce d'en neutraliser la portée. Puisque, selon les termes de l'apôtre Paul, «Christ est la fin de la Loi» (Rom. X, 4), celle-ci est devenue caduque (sauf pour les préceptes du Décalogue, qui formulent les exigences de la loi naturelle). De plus, les textes du Nouveau Testament sont parfaitement clairs : le royaume du Christ n'appartient pas à ce monde (Jean XVIII, 36) et les chrétiens sont tenus de se soumettre aux pouvoirs publics, donc à des empereurs notoirement païens et même persécuteurs des fidèles, ce qui implique la légitimité de leur fonction, considérée en elle-même et hors de toute référence religieuse (Rom. XIII, 1-7).

\section{Une argumentation théologique}

Cette argumentation théologique est fondée sur la dispensation ou, comme disent les théologiens, sur «l'économie» du plan salvifique de Dieu. Certes, il est évident, selon l'Écriture, que la loi de Moïse, ou l'ancienne Alliance n'a existé que pour mener l'humanité jusqu'au Christ et à son œuvre de salut. Mais peut-on dire que l'ordre de la création, avec son complément, le droit naturel, ont été voulus par Dieu comme un moyen en vue du Christ? Nullement, répond Amyraut en bon calviniste. La chute

18. Bayle, 1734, vol. I, p. 272-273, note P. 
d'Adam est un énorme accident de l'histoire, dû à la seule faute de l'homme et toute la dispensation du salut, présentée dans le Nouveau Testament, n'est là que pour réparer les dégâts. Cette axiomatique théologique comporte une immédiate conséquence politique : le plan primitif de Dieu, manifesté dans l'œuvre créatrice, est antérieur et supérieur à l'ordre de la grâce. Donc aucun roi ou magistrat ne peut, au nom des intérêts de la foi, s'élever contre les stipulations du droit naturel, qui prescrit aux sociétés humaines de vivre en paix et tranquillité. Plus précisément même, Amyraut, considérant que tous les hommes naissent libres, mais qu'ils remettent leur liberté personnelle entre les mains du souverain chargé du bon ordre, explique que le fondement des droits des sujets dans le royaume est triple : la nature, la police (l'ensemble des constitutions et des lois), la religion. Celle-ci ne vient qu'en dernier lieu. «Le troisième [droit] finalement est celuy de la Religion, qui ne sçauroit subsister si celuy de la Nature et de la Police ne demeurent. Car pour ce que nous sommes hommes avant que d'être chrestiens, les sentimens de la Nature précèdent en nous les créances de la Foy ${ }^{19}$.»

La subordination des droits religieux à ceux que fondent la « Nature » et la «Police» conduit Amyraut à faire deux parts dans les actions du monarque comme dans celles des magistrats et des officiers. En tant que détenteur de la puissance publique, le souverain et ses subordonnés ne peuvent que faire appliquer la Police du royaume, elle-même réglée sur le droit naturel. Comme celui-ci impose de faire tout ce qui favorise la vie en commun, le devoir des détenteurs de la puissance politique est en France de faire appliquer l'édit de Nantes, comme n'importe quelle autre décision de justice (par exemple, celle qui concerne la dévolution d'un héritage, les limites d'une propriété, etc.). Le fait que la décision s'applique à des droits concernant l'exercice du culte ne change rien à la nature du juste et de l'injuste. En revanche, comme personne privée - Amyraut écrit : en son particulier —, le roi ou chacun de ses officiers a non seulement le droit, mais même le devoir de travailler au développement de sa confession. Seulement, ce zèle ne doit pas être déployé par le détenteur de l'autorité politique en tant que tel, car, en favorisant une confession au détriment d'une autre par le moyen de «la loy publique», il agirait injustement.

Si nous revenons maintenant vers les trois autres argumentaires développés en faveur de la tolérance civile des religions, nous constatons, d'une part, que la doctrine des protestants saumurois est sans doute la plus satisfaisante au point de vue théologique, car elle préserve la propriété de vérité à laquelle tient fermement la théologie chrétienne, et, d'autre part, qu'elle ne subordonne pas la puissance spirituelle à la puissance politique. Certes,

19. Аmyraut, 1647, p. 43. 
Amyraut ne craint pas de reprendre plusieurs fois à son compte le mot de Lucrèce, «Quantum potuit religio suadere malorum! ». Mais le remède au fanatisme ne consiste pas, selon lui, à faire diriger l'Église par le prince. Pour lui, en effet, le ministère de l'Église n'est pas acquis par délégation de la souveraineté temporelle, il représente sacramentellement celui du Christ et appartient donc à la souveraineté de celui-ci. Mais il faut que chacun reste chez soi, le souverain veillant à ce que Dieu soit servi selon la conscience de chacun (s'il existe dans le royaume plusieurs confessions), les ministres de la Parole rappelant le souverain à se conformer dans l'exercice de sa charge aux préceptes divins. La création avant la révélation, la nature avant la grâce, la stabilité de la société avant l'exigence de l'unité religieuse. Cette axiomatique théologique apporte une mutation théorique dans le champ du politique, puisqu'elle introduit la priorité du droit naturel sur toute autre disposition juridique. La fin $\mathrm{du}_{\mathrm{XVII}}{ }^{\mathrm{e}}$ siècle et le siècle des Lumières tout entier allaient assister à la prolifération de traités sur le droit naturel. C'est en s'appuyant sur lui que les juristes favorables à l'attribution d'un état civil aux sujets protestants du roi de France obtiendront l'édit de tolérance de 1786.

Cet appel au droit naturel n'était pas entièrement nouveau. Déjà, au $\mathrm{XIV}^{\mathrm{e}}$ siècle, des théologiens s'étaient élevés contre les prétentions théocratiques de la papauté en affirmant une certaine autonomie du temporel. Par exemple, Jean Quidort, dit Jean de Paris, Pierre de La Palu et Durand de Saint-Pourçain :

«Soulignant la distinction entre le domaine naturel et le domaine surnaturel, ils affirment que le regnum n'est pas le fruit du péché et ne s'occupe pas que des corps, mais découle directement de Dieu par droit naturel [Jean Quidort] ou du peuple qui le délègue au souverain [Pierre de La Palu], ce qu'admettait également un autre théologien dominicain, Durand de Saint-Pourçain ${ }^{20}$. »

$\mathrm{Au} \mathrm{XVI}^{\mathrm{e}}$ siècle, ce sont aussi les théologiens dominicains comme Francisco de Vitoria et Bartolomé de Las Cases qui affirment que le pape n'est nullement le souverain temporel du monde. Ces enseignements se conforment à celui de saint Thomas d'Aquin, mais c'est sans doute à travers la scolastique espagnole $\mathrm{du} \mathrm{XvI}^{\mathrm{e}}$ siècle, influente même parmi les théologiens protestants, qu'a pu s'effectuer la surprenante rencontre entre le thomisme et la doctrine politique d'Amyraut.

François LAPLANCHE (juillet 2001).

20. Vauchez, 1990, p. 280. 


\section{LISTE DES RÉFÉRENCES}

Amyraut (Moyse), 1647, Apologie pour ceux de la religion, Saumur, Lesnier. Bayle (Pierre), 1734, Dictionnaire historique et critique, $1^{\text {re }}$ éd. 1695-1697, ici $5^{\mathrm{e}}$ éd. Amsterdam, 5 vol.

Bots (Hans) et Leroy (Pierre), 1978, Correspondance intégrale d'André Rivet et de Claude Sarrau. T. I : La République des lettres à la fin du règne de Louis XIII (septembre 1641-mai 1643), Amsterdam/Maarssen, Academic Publishers Associated/Holland University Press.

CARBonier-Burkard (Marianne), 1998, «Les préambules des édits de pacification », Bulletin de la Société de l'histoire du protestantisme français, t. CXLIV, p. 75-92.

Duplessis-Mornay (Philippe), 1824-1825, Mémoires et correspondance de Duplessis-Mornay, publ. par A. D. De LA Fontenelle de Vaudoré et P. R. Auguis, Paris, Treuttel et Würtz, 12 vol.

Garrisson (Janine), 1998, L'Édit de Nantes, Paris, Fayard.

Labrousse (Élisabeth), 1985, «Une foi, une loi, un roi? » Essai sur la révocation de l'édit de Nantes, Paris/Genève, Payot/Labor et Fides.

Lagrée (Jacqueline), 1989, Le Salut du laïc. Sur Herbert de Cherbury, Paris, Vrin.

LAGRÉE (J.), 1991, La Raison ardente. Religion naturelle et raison au XVII siècle, Paris, Vrin.

LAPlanche (François), 1955, Orthodoxie et prédication. L'œuvre d'Amyraut et la querelle de la grâce universelle, Paris, Presses universitaires de France.

LaPlanche (F.), 1986, L'Écriture, le sacré et l'histoire. Érudits et politiques protestants devant la Bible en France au XvII siècle, Amsterdam/Maarssen, Academic Publishers Associated/Holland University Press.

LeCler (Joseph), 1955, Histoire de la tolérance au siècle de la Réforme, Paris, Aubier, 2 vol.

Locke (John), 1980, Lettre sur la tolérance, Genève, Slatkine Reprints.

Mousnier (Roland), 1964, L'Assassinat d'Henri IV, Paris, Gallimard (Trente journées qui ont fait la France, 13).

PanNIER (Jacques), 1931, L'Église réformée de Paris sous Louis XIII (16211629), Paris, Honoré Champion, 2 vol.

Thuau (Étienne), 1966, Raison d'État et pensée politique à l'époque de Richelieu, Paris, Armand Colin.

VAuchez (André), 1990, «L'idée d'Église dans l'Occident latin », in Histoire du christianisme. Vol. VI : Un temps d'épreuves (1274-1449), Paris, Desclée de Brouwer. 